\title{
Resonant transmission near nonrobust periodic slab modes
}

\author{
Stephen P. Shipman* \\ Department of Mathematics, Louisiana State University, Lockett Hall 304, Baton Rouge, Louisiana 70803, USA \\ Stephanos Venakides ${ }^{\dagger}$ \\ Department of Mathematics, Duke University, Durham, North Carolina 27708, USA \\ (Received 17 December 2003; revised manuscript received 30 August 2004; published 23 February 2005)
}

\begin{abstract}
We present a precise theoretical explanation and prediction of certain resonant peaks and dips in the electromagnetic transmission coefficient of periodically structured slabs in the presence of nonrobust guided slab modes. We also derive the leading asymptotic behavior of the related phenomenon of resonant enhancement near the guided mode. The theory applies to structures in which losses are negligible and to very general geometries of the unit cell. It is based on boundary-integral representations of the electromagnetic fields. These depend on the frequency and on the Bloch wave vector and provide a complex-analytic connection in these parameters between generalized scattering states and guided slab modes. The perturbation of three coincident zeros-those of the dispersion relation for slab modes, the reflection constant, and the transmission constant-is central to calculating transmission anomalies both for lossless dielectric materials and for perfect metals.
\end{abstract}

DOI: $10.1103 /$ PhysRevE.71.026611

\section{INTRODUCTION}

The phenomenon of resonant transmission and reflection of electromagnetic energy by periodically structured films or slabs, at wavelengths on the order of or longer than the structural periodicity, occurs in various types of materials, from dielectrics to metals [1-9]. It is known to be intimately connected with the waveguide resonances, or modes, of the periodic structure and resonant enhancement of incident source fields in the presence of modes. In metal films, these resonances are surface plasmons; their connection to extraordinary transmission is a relatively recent discovery [1] and has sparked renewed interest in controlling these phenomena, which are recognized to hold promise in the design of photonic devices involving filters, lasers, and integrated optics $[2-4,10]$.

Various explanations have been offered for this connection. In the case of surface plasmons in periodically structured metal slabs, as gratings or films with holes, it is observed that the coupling of plasmons on both sides of the film facilitates the transmission of incident fields [3,5]; it is also argued that the excited plasmons and the transmitted field are two inherently related aspects of the phenomenon of resonant scattering by a periodic structure [6]. See [11] for a survey of some of the literature. It is long known that transmission peaks are closely associated with high reflectivity conditions at the interface of a solid dielectric slab with the surrounding medium (as air) (e.g., [12]). Structural periodicity enhances these effects, and particularly pronounced resonant enhancement and sharp transmission peaks and dips occur in the presence of structural defects in the underlying periodicity $[8,9]$.

\footnotetext{
*Email address: shipman@math.lsu.edu

†Email address: ven@math.duke.edu
}

PACS number(s): 42.70.Qs, 41.20.Jb, 42.25.Fx, 42.79.Dj

Our present work is motivated by a transmission anomaly that we observed numerically in a periodic arrangement of dielectric rods [9] near the wave number and frequency of a bound slab mode, or bound state. (Figs. 2, 3, and 5). The bound state occurs at the lowest resonant frequency of the structure. We characterize it as nonrobust because it disappears under perturbation of the Bloch wave vector. This perturbation produces sharp downward and upward spikes in the transmission coefficient viewed as a function of frequency; the spikes emanate from the frequency of the bound state, becoming less sharp as the perturbation grows. This work (Sec. II) provides a simple theoretical formula for the transmission coefficient as a function of the wave number and frequency near the bound state. The formula makes a sharp quantitative prediction of the anomaly with only the knowledge of four experimentally (or numerically) determined values [Eq. (12) and Fig. 3]. These values depend on the specific geometry and electromagnetic properties of the structure.

The transmission anomaly is accompanied by significant resonant enhancement of plane-wave source fields at frequencies in the region of the spikes (Fig. 5), where resonant fields appear in the body of the periodic structure. Our theory produces (Sec. III) formulas for the measure of the field enhancement as a function of wave number and frequency near the bound state and is in good agreement with numerical results (Fig. 6). Again, the formulas contain parameters that depend on the particular nature of the slab structure.

The anomaly occurs because three zeros, namely those of an eigenvalue describing the dispersion relation for slab modes, a complex reflection amplitude, and a complex transmission amplitude, that are coincident at the bound state, split apart continuously upon perturbation of the wave number. Our analysis relies on the fact that these functions depend analytically on the wave number and frequency.

An advantage of our approach is that it is independent of the specific geometry of the structure and independent of 


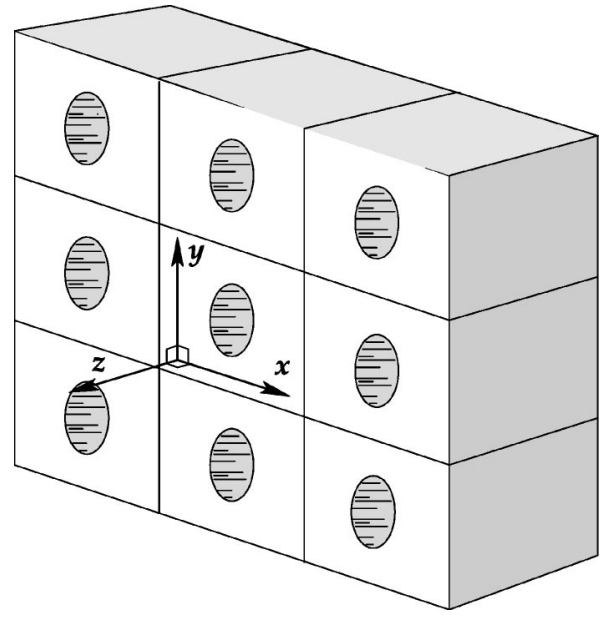

FIG. 1. A three-dimensional two-phase periodic slab. The structure continues periodically in the $x$ and $y$ directions.

whether the structure is a perfect metal (in which case the phenomenon is mediated by plasmons) or a lossless dielectric. Indeed, the theory applies to a general class of periodically structured slabs (e.g., Figs. 1 and 2) which display a nonrobust bound state. These include lossless dielectrics as well as metals for which the approximation by a perfect conductor boundary condition is valid.

The resonance behavior we describe is not related to the Wood anomaly, which is known to coincide with certain minima in transmission through metal films [7]. The Wood anomaly occurs at cutoff wave numbers (or frequencies) of the propagating Fourier (Bragg) harmonics, where the complex analyticity of the fundamental solution of the Maxwell equations fails.

In this work, we focus on calculations for twodimensional structures (those that are constant in one space direction, as in Fig. 2) and compare our theory with numerical results. In future communication, we will show how the analysis applies to three-dimensional structures, and we will extend our present results to include anomalies due to perturbations in the geometry of the periodic structure, such as surface and channel defects, which we investigated numerically in $[9,13]$. In principle, the theory is not restricted to

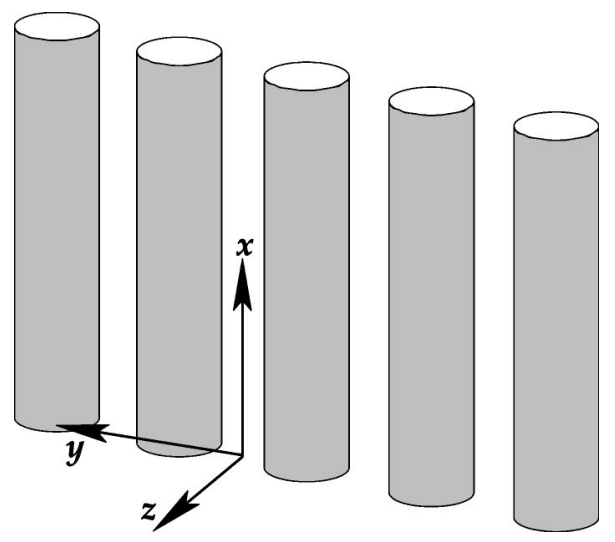

FIG. 2. A two-dimensional periodic slab. The structure continues constantly in the $x$ direction and periodically in the $y$ direction.
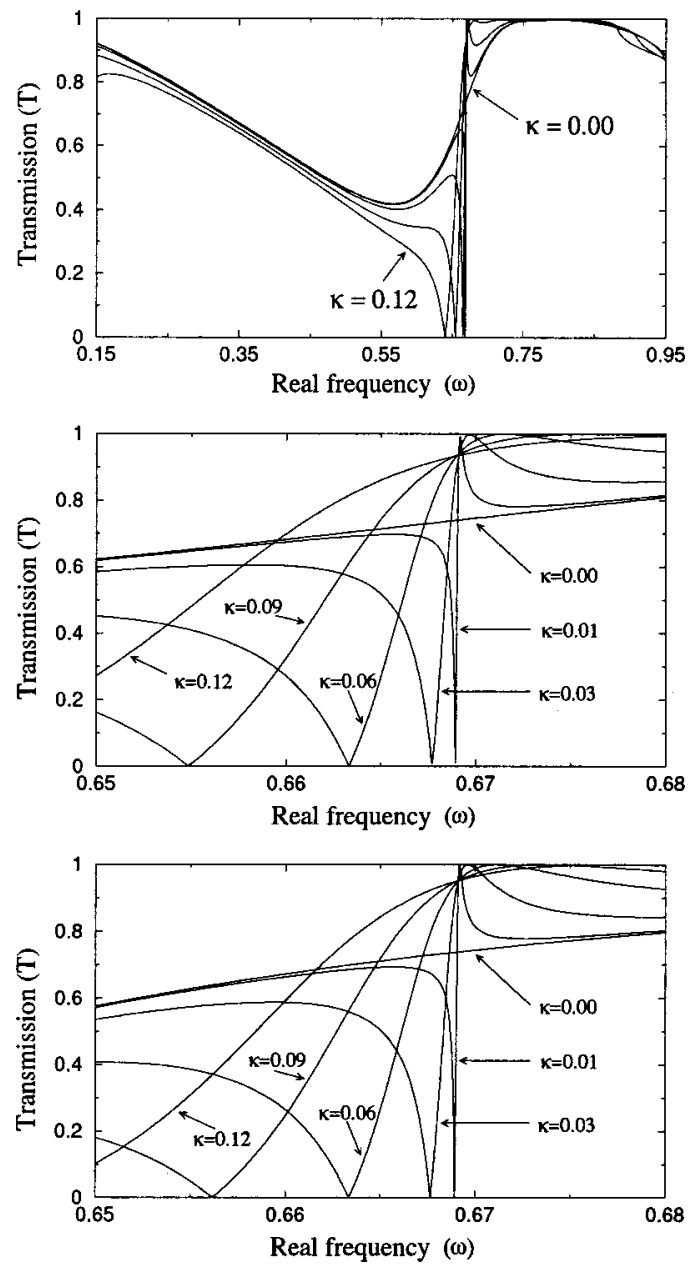

FIG. 3. Upper: Numerical simulation, by boundary integral equations, of transmission vs reduced frequency for electrically polarized plane-wave source fields through a slab of vertical rods in air (Fig. 2) for various values of the wave number $\kappa$ in the $y$ direction. The dielectric contrast is 12 , and the magnetic permeability is 1. $T$ is the square root of the proportion of the source energy that is transmitted. Middle: A closer view of the top figure near the region of anomalous transmission. Lower: Theoretical prediction of anomalies. In formula (12), $t_{0} \approx 0.739, t \approx 1.6, r \approx-0.56$, and $\eta$ $\approx 18.69$ were estimated from the numerical simulations.

electromagnetics, but extends to similar phenomena in acoustics and elasticity.

\section{TRANSMISSION ANOMALIES}

We discuss nonrobust bound slab modes and describe the asymptotics of the dispersion relation and the scattering problem near such a mode; then we derive the main result, which is a theoretical prediction of the behavior of anomalous transmission near a nonrobust mode [Eq. (12) and Fig. $3]$. Details of the supporting mathematical theory are given in the Appendixes.

We work with the Maxwell equations at constant (complex) frequencies $\omega$. The fields we deal with are timeindependent and become full Maxwell solutions when multiplied by the exponential time-dependent factor $e^{-i \omega t}$. The 
periodic slab structures are of a homogeneous material contrasting from the ambient material exterior to the slab. Our main analytic tool is a system of integral equations arising from the Maxwell equations and given in detail in Appendix B. They are written symbolically as

$$
\mathrm{A} \psi=\phi
$$

and given in full form in Eq. (B3) for perfect metals and Eqs. (B4) for dielectrics. Here, $\boldsymbol{\phi}$ represents the trace (tangential component) of the electric and magnetic source fields on the interface between the material of the slab and the material exterior to it within a period cell of the structure, and $\psi$ represents the trace of the external electric and magnetic fields on the same interface. The external field is the sum of the source field and the scattered field. The bounded linear integral operator $\mathrm{A}=\mathrm{A}(\boldsymbol{\kappa}, \omega)$ has an analytic dependence on the Bloch wave vector $\boldsymbol{\kappa}$ and the frequency $\omega$ in our $(\boldsymbol{\kappa}, \omega)$ region of interest. Throughout, $\omega$ and $\boldsymbol{\kappa}$ represent nondimensionalized quantities, and the (nondimensionalized) period of the unit cell is taken to be $2 \pi$. $^{1}$

We present our calculations in a two-dimensional reduction of the problem, that is, we assume that the slab structure and electromagnetic fields are constant in the $x$ direction (Fig. 2). In this case, the Bloch wave vector has a component only in the $y$ direction, which we denote by $\kappa$. The fields decouple into the two primary polarizations, and the harmonic Maxwell equations reduce to the scalar Helmholtz equation for the field component directed out of the $y z$ plane, in the $x$ direction. The source and total field traces $(\boldsymbol{\phi}$ and $\psi$ ) on the slab interface represent the field value and its normal derivative. The integral equation $\mathrm{A} \boldsymbol{\psi} \boldsymbol{\phi}=\boldsymbol{\phi}$ reduces to Eqs. (B6). If the trace of the field is known, the full external field is calculated directly from Green's identity (B7) below, in which the outgoing Helmholtz Green's function (B5) corresponds to the periodic structure of our problem. A field satisfies the outgoing condition if, for large values of $|z|$, it has an expansion in Fourier harmonics,

$$
\psi=\sum_{m=-\infty}^{\infty} c_{m}^{ \pm} e^{i(m+\kappa) y} e^{ \pm \gamma_{m} z},
$$

for sufficiently large values of $|z|$. The + and - signs refer to $z$ being large and positive and $z$ being large and negative, respectively, the $c_{m}$ are constants, and $\gamma_{m}=\left[-\epsilon_{0} \mu_{0} \omega^{2}+(m\right.$ $\left.+\kappa)^{2}\right]^{1 / 2} \neq 0 . \epsilon_{0}$ and $\mu_{0}$ are the dielectric and magnetic constants exterior to the slab. We will assume that they are both equal to 1 . In this theory, we are interested in real values of $\kappa$, and we assume that $\kappa$ lies in the first Brillouin zone $(|\kappa|$ $<1 / 2$ ). When $\omega$ is real and $|\omega|<|\kappa|$, which is the region outside the light cone, all the exponentials decay as $|z| \rightarrow \infty$. The region of interest for us is the region inside the light

\footnotetext{
${ }^{1}$ If $L$ is the physical length of a period cell, $X$ denotes a physical three-dimensional length vector, and $T$ denotes physical time, then the nondimensionalized space and time variables are $(x, y, z)=\boldsymbol{x}$ $=(2 \pi / L) \boldsymbol{X}$ and $t=(2 \pi c / L) T$, where $c$ is the speed of light in a vacuum. If $f$ is the frequency (cycles per time) and $k$ is the wave number (cycles per length), then the nondimensionalized wave number and frequency are $|\boldsymbol{\kappa}|=k L$ and $\omega=f L / c$.
}

cone where all the exponentials decay except for those corresponding to $m=0$ (one for $z>0$ and one for $z<0$ ), which are plane-wave propagating harmonics. This is the region where $|\kappa|<\omega<|\kappa+m|$ for all integers $m \neq 0$.

A bound state is a field $\psi$ that decays as $|z| \rightarrow \infty$ and has a trace $\boldsymbol{\psi}$ on the material interface that satisfies the sourceless equation

$$
\mathrm{A} \psi \boldsymbol{\psi}=\mathbf{0}
$$

By an argument that relies on conservation of energy, one may prove that bound states occur only at real frequencies and wave numbers. Typically, a robust bound state occurs at a $(\kappa, \omega)$ pair for which Green's function $G$ for the Helmholtz equation has no Fourier harmonics that propagate in $z$, so that $G$ decays as $|z| \rightarrow \infty$. This is the region outside the light cone for the exterior medium for values of $\kappa$ in the first Brillouin zone, $|\kappa|<1 / 2$. In this case, real perturbations of $\kappa$ result in real perturbations of the value of $\omega$ for which a bound state exists; in other words, there is a local real dispersion relation $\omega=W(\kappa)$. At values of $(\kappa, \omega)$ that do admit propagating harmonics (as in our case), bound slab modes are generally precluded because these harmonics carry energy away from the slab. Under certain symmetry conditions (typically at $\kappa=0$ ), there exist states whose Fourier decomposition contains none of the propagating harmonics (the corresponding constants $c_{m}$ are zero); the energy is therefore bound to the slab. Upon a real perturbation of $\kappa$ in such a bound state, the symmetry of the field is broken, causing the bound state to disappear. Frequencies $\omega=W(\kappa)$ at which the sourceless equation $\mathrm{A} \boldsymbol{\psi}=\mathbf{0}$ is still solvable acquire nonzero imaginary parts, i.e., the dispersion relation becomes complex. Necessarily, the exponents $\gamma_{m}$ of the propagating harmonics also acquire nonzero imaginary parts, and the corresponding exponentials now have growth in $|z|$. Such a bound state is nonrobust. Nonrobust bound states correspond to eigenvalues embedded in the continuous spectrum of the Helmholtz operator for the entire structure; they are known to exist also in acoustic waveguides [14].

To summarize, the solutions of the sourceless problem $\mathrm{A} \boldsymbol{\psi}=\mathbf{0}$ occur at values of $\kappa$ and $\omega$ where the operator A has a zero eigenvalue $\ell=\ell(\kappa, \omega)=0$. The relation $\ell(\kappa, \omega)=0$ or $\omega=W(\kappa)$ when solved for $\omega$ is the dispersion relation. When the full field corresponding to the sourceless trace $\psi$ decays away from the structure (as $|z| \rightarrow \infty$ ), the field defines a strict guided slab mode, a bound state. When that field fails to decay, the corresponding pair $(\kappa, \omega=W(\kappa))$ is called a resonance. Bound states occur at real pairs $(\kappa, \omega)$ that satisfy the dispersion relation; they are nonrobust when real perturbations of $\kappa$ turn them into resonances, that is, when $\omega$ $=W(\kappa)$ acquires a nonzero imaginary part. Resonances correspond to what are often referred to as leaky modes (see, e.g., $[8,12])$.

We analyze nonrobust bound states that correspond to a simple zero eigenvalue $\ell$ (that is, having multiplicity 1) occurring at $\kappa=0$ and $\omega=\omega_{0}>0$. The imaginary part of $\omega$ for real values of $\kappa$ cannot be positive (as the corresponding time-harmonic Maxwell field would be growing in time) [9]. Consequently the simplest form for a local solution of 


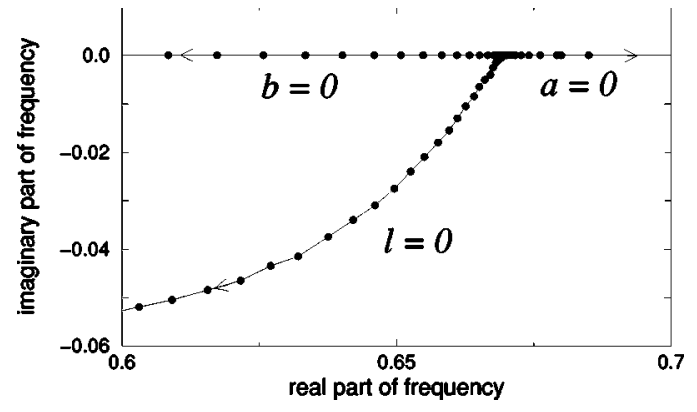

FIG. 4. The dispersion relation $\ell=0$ and the zero sets $a=0$ and $b=0$ of the reflection and transmission constants for real values of $\kappa$. The solid points represent numerically calculated values of frequency, plotted in the complex $\omega$ plane, for values of $\kappa$ ranging from 0.0 to about 0.20 , where $\ell(\kappa, \omega)=0, a(\kappa, \omega)=0$, and $b(\kappa, \omega)$ $=0$. The point in common is at $\kappa=0$, where the bound state occurs.

$\ell(\kappa, \omega)=0$, in which $\omega$ is expressible as a power series in $\kappa$, is

$$
\ell(\kappa, \omega)=0 \Leftrightarrow \omega=\omega_{0}+c \kappa+s \kappa^{2}+\mathcal{T}_{\mathrm{HO}}(\kappa),
$$

where $\mathcal{T}_{\text {HO }}$ stands for higher-order terms. $c$ is real, and the imaginary part of $s$ is nonpositive because the imaginary part of $\omega$ is not allowed to be positive. Our theory examines the case $c=0$. This is forced, for example, by any symmetry that requires the dispersion relation to be an even function of the wave number, as is the case of the structure of Fig. 2. Thus,

$$
\ell(\kappa, \omega)=0 \Leftrightarrow \omega=\omega_{0}+s \kappa^{2}+\mathcal{T}_{\mathrm{HO}}(\kappa),
$$

in which we make the nondegeneracy assumption that Im $(s) \neq 0$. This causes the dispersion relation for real $\kappa$ to enter the lower half $\omega$ plane transversely to the real $\omega$ axis (as seen in Fig. 4).

For values of $(\kappa, \omega)$ for which $\ell(\kappa, \omega) \neq 0$, we introduce a plane-wave source field $\phi$ and normalize its amplitude by the eigenvalue $\ell$,

$$
\ell \phi=\ell e^{i(\kappa y+\gamma z)} e^{-i \omega t},
$$

where $\gamma=\sqrt{\omega^{2}-\kappa^{2}}$. The integral equation is uniquely solvable and the full field corresponding to its solution satisfies the asymptotic relation

$$
\begin{gathered}
\psi \sim\left(\ell e^{i \gamma z}+a e^{-i \gamma z}\right) e^{i \kappa y}(z \rightarrow-\infty), \\
\psi \sim b e^{i \gamma z} e^{i \kappa y}(z \rightarrow \infty) .
\end{gathered}
$$

In this expression, $a(\kappa, \omega)$ is the reflected complex amplitude and $b(\kappa, \omega)$ is the transmitted amplitude.

The crucial point behind our explanation of the transmission anomaly is that both coefficients $a$ and $b$ can be extended in the complex variables $\kappa$ and $\omega$ into the relation $\ell(\kappa, \omega)=0$ and are analytic in a complex neighborhood of the point $\left(0, \omega_{0}\right)$. (Proof of these statements is given in the remark and in the analysis preceding it in Sec. III). Moreover, since $\ell$ is zero at $\left(0, \omega_{0}\right)$, both $a$ and $b$ must be zero there. As $\kappa$ is perturbed from 0 taking real values, the coinciding zeros of $\ell, a$, and $b$ separate continuously in the complex $\omega$ plane (Fig. 4). The upward spike seen in Fig. 3 occurs
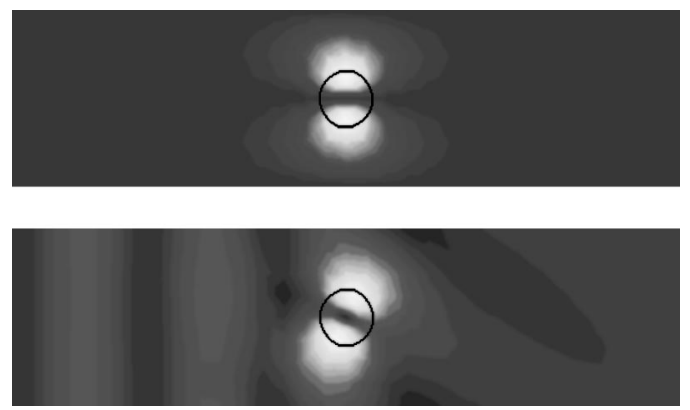

FIG. 5. Upper: A contour plot of the amplitude of the bound state at $\kappa=0.0$ and $\omega \approx 0.669$. Lower: A resonant field produced by an incident plane wave at $\kappa=0.12$ and $\omega=0.660$. A cross section of one period of the structure of Fig. 2 is shown, with the boundary of the rod indicated artificially by the black circle. The $y$ axis is vertical, the $z$ axis is horizontal, and the $x$ axis is out of the page. White indicates maximal amplitude, while black indicates zero amplitude.

at the frequency satisfying $a(\kappa, \omega)=0$ that corresponds to $100 \%$ transmission, while the accompanying deep dip occurs at the frequency satisfying $b(\kappa, \omega)=0$, which forces zero transmission. These statements are, of course, true only up to numerical resolution.

We have tested our results on the two-dimensional highcontrast dielectric numerical example in Figs. 2-5. The structure is an infinite row of rods of radius 1 standing parallel to the $x$ axis and separated periodically by $2 \pi$ in the $y$ direction, as in Fig. 2. The interior dielectric coefficient is 12, the exterior 1 , and both magnetic coefficients are 1 . We find numerically that there exists a bound guided mode at $\left(\kappa=\kappa_{0}\right.$ $\left.=0, \omega=\omega_{0} \approx 0.669\right)$. It is $E$-polarized, that is, the electric field points in the $x$ direction in Fig. 2. The eigenvalue $\ell$ has multiplicity 1 in the vicinity of $\left(0, \omega_{0}\right)$, and $\ell\left(0, \omega_{0}\right)=0$. The guided mode is actually a standing wave that is supported by the slab, as $\kappa=0$ and $\omega$ is real; it decays as $|z| \rightarrow \infty$ (Fig. 5). In a complex neighborhood of $\left(0, \omega_{0}\right)$, the Green's function [Eq. (B5)] for the Helmholtz equation possesses only one propagating harmonic $(m=0)$ at $\left(0, \omega_{0}\right)$, and therefore the total field resulting from scattering of the source $\ell \phi$ is in fact characterized far away from the slab by a single reflection amplitude $a$ and a single transmission amplitude $b$, as described above. As $|\ell|^{2}=|a|^{2}+|b|^{2}$ for real $(\kappa, \omega)$, it follows that $a\left(0, \omega_{0}\right)=b\left(0, \omega_{0}\right)=0$.

The techniques discussed are applicable to general periodic slab structures (including three-dimensional structures) in which energy is conserved. The only additional requirements on our structure so far have been a simple nonrobust bound state at $(\kappa, \omega)=\left(0, \omega_{0}\right)$ and the condition $c=0$ in the dispersion relation; both are typically forced by some symmetry. We also make the generic assumptions that $\partial a / \partial \omega\left(0, \omega_{0}\right) \neq 0$ and $\partial b / \partial \omega\left(0, \omega_{0}\right) \neq 0$.

In the ensuing analysis, we let $\varpi=\omega-\omega_{0}$ in Eq. (1). The Weierstraß preparation theorem for analytic functions of two variables (see, for example, [15]) dictates the following forms for $\ell, a$, and $b$ :

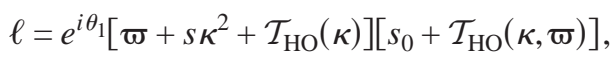




$$
\begin{aligned}
& a=e^{i \theta_{2}}\left[\varpi+r_{1} \kappa+r \kappa^{2}+\mathcal{T}_{\mathrm{HO}}(\kappa)\right]\left[r_{0}+\mathcal{T}_{\mathrm{HO}}(\kappa, \varpi)\right], \\
& b=e^{i \theta_{3}}\left[\varpi+t_{1} \kappa+t \kappa^{2}+\mathcal{T}_{\mathrm{HO}}(\kappa)\right]\left[t_{0}+\mathcal{T}_{\mathrm{HO}}(\kappa, \boldsymbol{\varpi})\right],
\end{aligned}
$$

where $s_{0}, r_{0}$, and $t_{0}$ are positive real numbers. Inserting these forms into the relation $|\ell|^{2}=|a|^{2}+|b|^{2}$ for real $(\kappa, \boldsymbol{\varpi})$ and matching like terms yields the relations

$$
\begin{gathered}
r_{0}^{2}+t_{0}^{2}=s_{0}^{2}\left(\varpi^{2} \text { term }\right), \\
\left|r_{0} r_{1}\right|^{2}+\left|t_{0} t_{1}\right|^{2}=0\left(\kappa^{2} \text { term }\right),
\end{gathered}
$$

which implies $r_{1}=t_{1}=0$, and using this, we obtain $\operatorname{Re}[(s)$ denotes the real part of $s$ ]

$$
\begin{gathered}
\operatorname{Re}(s)=r_{0}^{2} \operatorname{Re}(r)+t_{0}^{2} \operatorname{Re}(t)\left(\varpi \kappa^{2} \text { term }\right), \\
|s|^{2}=r_{0}^{2}|r|^{2}+t_{0}^{2}|t|^{2}\left(\kappa^{4} \text { term }\right) .
\end{gathered}
$$

We thus arrive at the expressions

$$
\begin{aligned}
& \ell=e^{i \theta_{1}}\left[\varpi+s \kappa^{2}+\mathcal{T}_{\mathrm{HO}}(\kappa)\right]\left[s_{0}+\mathcal{T}_{\mathrm{HO}}(\kappa, \varpi)\right], \\
& a=e^{i \theta_{2}}\left[\varpi+r \kappa^{2}+\mathcal{T}_{\mathrm{HO}}(\kappa)\right]\left[r_{0}+\mathcal{T}_{\mathrm{HO}}(\kappa, \varpi)\right], \\
& b=e^{i \theta_{3}}\left[\varpi+t \kappa^{2}+\mathcal{T}_{\mathrm{HO}}(\kappa)\right]\left[t_{0}+\mathcal{T}_{\mathrm{HO}}(\kappa, \varpi)\right],
\end{aligned}
$$

and the following relations for complex pairs near $\left(0, \omega_{0}\right)$ :

$$
\begin{aligned}
& \ell=0 \Leftrightarrow \omega=\omega_{0}-s \kappa^{2}+\mathcal{T}_{\mathrm{HO}}(\kappa), \\
& a=0 \Leftrightarrow \omega=\omega_{0}-r \kappa^{2}+\mathcal{T}_{\mathrm{HO}}(\kappa), \\
& b=0 \Leftrightarrow \omega=\omega_{0}-t \kappa^{2}+\mathcal{T}_{\mathrm{HO}}(\kappa) .
\end{aligned}
$$

Due to the analyticity in $\kappa$ and $\omega$, these expressions are valid also for $(\kappa, \omega)$ in a complex neighborhood of $\left(0, \omega_{0}\right)$. Because of Eqs. (2) and (4), $\operatorname{Re}(s)$ lies between $\operatorname{Re}(r)$ and $\operatorname{Re}(t)$, and, as long as these real parts are not equal, we deduce that, as the curve $\ell=0$ for real $\kappa$ emanates from the real $\omega$ axis, one of the curves $a=0$ or $b=0$ moves to its left and the other to its right in the complex $\omega$ plane (Fig. 4). If $\operatorname{Im}(r)=\operatorname{Im}(t)$ $=0$, then, to leading order, the curves $a=0$ and $b=0$ travel along the real $\omega$ axis, as $\kappa^{2} \geqslant 0$ increases, giving rise to two nearby values of $\omega$ moving apart from one another, one at which total reflection occurs $(b=0)$ and one at which total transmission occurs $(a=0)$. This is what happens in the numerical example, as we see the formation and spreading of the sharp dips and peaks in Fig. 3, although we do not have an analytic proof that $\operatorname{Im}(r)=\operatorname{Im}(t)=0$ in this specific case.

We show now how the knowledge of the three quantities $r$, $t$, and $t_{0}$ in Eqs. (7) and (8), as well as the slope (in $\omega$ ) of the transmission graph at the bound state, allow one to obtain a formula that approximates the transmission anomalies well. We include the first-order terms in the expressions for $a$ and $b$,

$$
\begin{gathered}
a=r_{0} e^{i \theta_{2}}\left(\varpi+r \kappa^{2}+\cdots\right)\left(1+r_{1} \varpi+r_{2} \kappa+\cdots\right), \\
b=t_{0} e^{i \theta_{3}}\left(\varpi+t \kappa^{2}+\cdots\right)\left(1+t_{1} \varpi+t_{2} \kappa+\cdots\right) .
\end{gathered}
$$

In the first factor, the higher-order terms are $\mathcal{O}\left(\kappa^{3}\right)$; in the second, they are $\mathcal{O}\left(\kappa^{2}+\varpi^{2}\right)$. The transmission coefficient $T$ (in the physics literature, the transmission coefficient is usually defined as our $T^{2}$ ) depends on the absolute value of the ratio $b / a$,

$$
T=\frac{|b|}{|\ell|}=\frac{|b|}{\sqrt{|a|^{2}+|b|^{2}}}=\frac{|b / a|}{\sqrt{1+|b / a|^{2}}},
$$

and $b / a$ has the form

$$
\frac{b}{a}=e^{i \theta} \frac{t_{0}\left(\varpi+t \kappa^{2}+\cdots\right)}{r_{0}\left(\varpi+r \kappa^{2}+\cdots\right)}\left(1+\eta_{1} \varpi+\eta_{2} \kappa+\cdots\right),
$$

in which $\theta=\theta_{3}-\theta_{2}, \eta_{1}=t_{1}-r_{1}$, and $\eta_{2}=t_{2}-r_{2} \cdot \eta_{1}$ and $\operatorname{Re}\left(\eta_{1}\right)$ have simple interpretations,

$$
\eta_{1}=\frac{r_{0}}{t_{0}} \frac{\partial(b / a)}{\partial \varpi}(0,0), \quad \operatorname{Re}\left(\eta_{1}\right)=\frac{r_{0}}{t_{0}} \frac{\partial|b / a|}{\partial \varpi}(0,0) .
$$

$[(0,0)$ refers to evaluation at $\kappa=0$ and $\varpi=0$.$] To put \operatorname{Re}\left(\eta_{1}\right)$ in terms of $T$, we use $r_{0}^{3} \partial|b / a| / \partial \varpi=\partial T / \partial \varpi$ at $\kappa=0$ and $\varpi$ $=0$,

$$
\eta:=\operatorname{Re}\left(\eta_{1}\right)=\frac{1}{t_{0} r_{0}^{2}} \frac{\partial T}{\partial \varpi}(0,0) .
$$

Whereas $\operatorname{Re}\left(\eta_{1}\right)$ has a clear meaning as an experimental value, the constant $\eta_{2}$ does not have such a simple interpretation. An accurate expression that captures the full asymptotic nature of the transmission anomaly depends on the real parts of both $\eta_{1}$ and $\eta_{2}$, as well as the $\kappa^{3}$ term in the first factors of $a$ and $b$ in Eqs. (9) and (10). Table I presents a detailed analysis of the asymptotic behavior of $b / a$ near the bound state in various asymptotic relations between $\kappa, \varpi$, and the characteristic ratio $\varpi / \kappa^{2}$, as $\kappa$ and $\varpi$ tend to zero. The column that shows the approximation of $b / a$ gives the first two terms in an asymptotic expansion in the various regimes. The $\kappa^{3}$ term in the first factors of $a$ and $b$ is significant only in the final row of the table, where it as well as $\eta_{2}$ affect the constant $\eta_{3}$.

Despite the complexity of the asymptotics and the difficulty in measuring $\eta_{2}$ and $\eta_{3}$, we demonstrate numerically that knowledge of $r, t, t_{0}$, and $\eta=\operatorname{Re}\left(\eta_{1}\right)$ alone $\left(r_{0}\right.$ is determined from $t_{0}$ by the relation $r_{0}^{2}+t_{0}^{2}=1$ ) delivers a good approximation of the transmission anomalies. Thus we use the approximation

$$
\left|\frac{b}{a}\right| \approx \frac{t_{0}\left|\varpi+t \kappa^{2}\right|}{r_{0}\left|\varpi+r \kappa^{2}\right|}(1+\eta \varpi),
$$

which is then substituted into expression (11) or $T$ to yield the result

$$
T \approx \frac{t_{0}\left|\varpi+t \kappa^{2}\right|(1+\eta \varpi)}{\sqrt{r_{0}^{2}\left|\varpi+r \kappa^{2}\right|^{2}+t_{0}^{2}\left|\varpi+t \kappa^{2}\right|^{2}(1+\eta \varpi)^{2}}} .
$$

Figure 3 shows a comparison between this approximation and the numerically calculated values of transmission in the region of the anomaly.

Table I (the fifth row) gives the first two terms of the asymptotic expansion for $b / a$ or $a / b$ in the $(\kappa, \omega)$ region near where $b=0$ or $a=0$, respectively. However, corrections for the error in the placement of the roots of $b$ and $a$, or the 
TABLE I. Asymptotics for the transmission anomaly.

\begin{tabular}{ccc}
\hline \hline $\begin{array}{c}\text { Asymptotic region } \\
\text { in the } \kappa \varpi \text { plane }\end{array}$ & $\begin{array}{c}\text { Corresponding relation } \\
\text { between } \kappa, \varpi, \text { and } \varpi / \kappa^{2}\end{array}$ & $\begin{array}{c}\text { Asymptotic value } \\
\text { of } b / a \text { or } a / b\end{array}$ \\
\hline$\kappa=0, \varpi \ll 1$ & $0=\kappa^{2} / \varpi=\kappa \ll \varpi \ll 1$ & $b / a \sim t_{0} / r_{0}\left(1+\eta_{1} \varpi\right)$ \\
$0 \neq \kappa \ll \varpi \ll 1$ & $\kappa^{2} / \varpi \ll \kappa \ll \varpi \ll 1$ & $b / a \sim t_{0} / r_{0}\left(1+\eta_{1} \varpi\right)$ \\
$\kappa \sim \varpi \ll 1$ & $\kappa^{2} / \varpi \ll \kappa \sim \varpi \ll 1$ & $b / a \sim t_{0} / r_{0}\left(1+\eta_{1} \varpi+\eta_{2} \kappa\right)$ \\
$\kappa^{2} \ll \varpi \ll \kappa \ll 1$ & $\varpi \ll \kappa \ll \kappa^{2} / \varpi \ll 1$ & $b / a \sim t_{0} / r_{0}\left(1+(t-r) \kappa^{2} / \varpi\right)$ \\
$\kappa^{2} \sim \varpi \ll \kappa \ll 1$ & $\varpi \ll \kappa \ll \kappa^{2} / \varpi \sim 1$ & $b / a \sim t_{0}\left(\varpi+t \kappa^{2}\right) / r_{0}\left(\varpi+r \kappa^{2}\right)\left(1+\eta_{2} \kappa\right)$ \\
$\kappa^{3} \ll \varpi \ll \kappa^{2} \ll 1$ & $a / b \sim r_{0}\left(\varpi+r \kappa^{2}\right) / t_{0}\left(\varpi+t \kappa^{2}\right)\left(1-\eta_{2} \kappa\right)$ \\
$\kappa^{3} \sim \varpi \ll \kappa^{2} \ll 1$ & $b / a \sim t_{0} t / r_{0} r\left(1+\left(t^{-1}-r^{-1}\right) \varpi / \kappa^{2}\right)$ \\
$0 \neq \varpi \ll \kappa^{3} \ll 1$ & $\varpi \ll \kappa \ll \varpi / \kappa^{2} \ll 1$ & $b / a \sim t_{0} t / r_{0} r\left(1+\left(t^{-1}-r^{-1}\right) \varpi / \kappa^{2}+\eta_{2} \kappa\right)$ \\
$\varpi=0, \kappa \ll 1$ & $\varpi \ll \kappa \sim \varpi / \kappa^{2} \ll 1$ & $b / a \sim t_{0} t / r_{0} r\left(1+\eta_{2} \kappa\right)$ \\
\hline \hline
\end{tabular}

placement of the points of $0 \%$ and $100 \%$ transmission, require knowledge of the $\kappa^{3}$ term in the first factors of $a$ and $b$. Indeed, in examining the middle and lower figures in Fig. 3, one can see an error in the point of zero transmission for $\kappa$ $=0.09$ that is roughly equal to $\kappa^{3}$.

As special asymptotic cases, we compute the limiting values of the transmission coefficient $T=|b / \ell|$ along the real $\kappa$ and $\omega$ axes,

$$
\begin{gathered}
\lim _{\omega \rightarrow \omega_{0}} T(0, \omega)=t_{0}, \\
\lim _{\kappa \rightarrow 0} T\left(\kappa, \omega_{0}\right)=t_{0}|t / s|=\frac{t_{0}|t|}{\sqrt{r_{0}^{2}|r|^{2}+t_{0}^{2}|t|^{2}}} .
\end{gathered}
$$

Because of Eqs. (2) and (5), $|s|$ lies between $|r|$ and $|t|$, and, as long as these moduli are not equal, we conclude that $|t|$ $\neq|s|$, so that $T$ is not continuous at $\left(0, \omega_{0}\right)$. From Fig. 3 , it is evident that the point of zero transmission moves away from $\omega_{0} \approx 0.669$ faster than the point of $100 \%$ transmission, as $\kappa$ increases from zero. This means that $|t|>|r|$, so that $|t|>|s|$, and the first limit in Eq. (13) is greater than the second. This behavior of the discontinuity in $T$ is observed in Fig. 3, where the transmission curves for small values of $\kappa$, as functions of $\omega$, come together near $\omega_{0}$ at a higher value than the value of the transmission curve for $\kappa=0$.

\section{RESONANT ENHANCEMENT}

We present a leading-order asymptotic theory to resonant field enhancement of plane-wave source fields scattered by periodic slab structures, a phenomenon that, according to our observations, accompanies anomalous transmission. The basic observation is summarized as follows. At normal incidence $(\kappa=0)$, the scattering problem for frequencies near that of the bound state $\left(\omega=\omega_{0}\right)$ exhibits no apparent anomalous behavior in numerical simulations: neither anomalous transmission nor resonant enhancement of plane-wave sources is observed. As $\kappa$ is perturbed from zero and the bound state disappears, fields of much higher amplitudes than the planewave source fields are observed in the slab structure at frequencies close to the double transmission "spike" that we discussed in the previous section. As $\kappa$ increases and the spike widens, the amount of "amplitude enhancement" decreases. The lower image in Fig. 5 shows a field produced by resonant scattering of a plane wave at a small value of $\kappa$ and a frequency $\omega$ near $\omega_{0}$.

Not surprisingly, the emerging high fields have a dominant contribution from the eigenfield of the operator A corresponding to the eigenvalue $\ell=\ell(\kappa, \omega)$. To understand this, we apply the spectral projection operator [16]

$$
\mathrm{P}_{1}=\frac{1}{2 \pi i} \oint_{\Gamma}\left(\ell^{\prime} \mathrm{I}-\mathrm{A}\right)^{-1} d \ell^{\prime}
$$

(the resolvent of $\mathrm{A}$ is integrated counterclockwise along a contour $\Gamma$ in the complex $\ell^{\prime}$ plane encircling the eigenvalue $\ell)$ to project any trace field $\boldsymbol{\psi} \in \mathcal{C}$ to the one-dimensional eigenspace corresponding to the eigenvalue $\ell=\ell(\kappa, \omega)$. In particular, applying the projection (at any value of $\kappa$ and $\omega$ ) to a fixed eigenvector $\hat{\psi}_{0}$ corresponding to $\kappa=\kappa_{0}=0$ and $\omega$ $=\omega_{0}($ where $\ell=0)$, we obtain the field

$$
\hat{\boldsymbol{\psi}}=\mathrm{P}_{1} \hat{\boldsymbol{\psi}}_{0}
$$

which is a basis for the one-dimensional eigenspace of $\mathrm{A}$ at $\ell$ and depends analytically on $\kappa$ and $\omega$.

The operator

$$
\mathrm{P}_{2}=\mathrm{I}-\mathrm{P}_{1}
$$

is also a projection and is complementary to $\mathrm{P}_{1}\left(\mathrm{P}_{1}+\mathrm{P}_{2}=\mathrm{I}\right)$. The images of these projections, denoted by $\mathcal{C}_{1}$ and $\mathcal{C}_{2}$, are independent subspaces of $\mathcal{C} . \mathcal{C}_{1}$ is the one-dimensional eigenspace of $\mathrm{A}$ for the eigenvalue $\ell(\kappa, \omega)$. A acts invariantly on $\mathcal{C}_{1}$ and on $\mathcal{C}_{2}$ and is therefore decomposed as $\mathrm{A}$ $=A_{1}+A_{2}$, where $A_{1}=A_{1}$ is multiplication by $\ell$ and $A_{2}$ $=\mathrm{AP}_{2}$ is a bounded invertible operator in $\mathcal{C}_{2}$.

Let now $\boldsymbol{\phi}$ be the trace of a source field $\phi$ that is analytic in $(\kappa, \omega)$ near a pair $\left(\kappa_{0}, \omega_{0}\right)$ where $\ell\left(\kappa_{0}, \omega_{0}\right)=0$. We demonstrate that our basic equation $\mathrm{A} \boldsymbol{\psi}=\ell \boldsymbol{\phi}$ has a solution that is analytic near $\left(\kappa_{0}, \omega_{0}\right)$ : Decompose the source field uniquely as $\boldsymbol{\phi}=\mathrm{P}_{1} \boldsymbol{\phi}+\mathrm{P}_{2} \boldsymbol{\phi}=\alpha \hat{\boldsymbol{\psi}}+\boldsymbol{\phi}_{2}$, where the complex scalar $\alpha$ and 
$\boldsymbol{\phi}_{2} \in \mathcal{C}_{2}$ are analytic. Then observe that $\boldsymbol{\psi}=\alpha \hat{\boldsymbol{\psi}}+\ell \mathrm{A}_{2}^{-1} \boldsymbol{\phi}_{2}$ is a solution. This can be seen clearly in matrix form, where one easily verifies

$$
\mathrm{A} \boldsymbol{\psi}=\left[\begin{array}{cc}
\ell & 0 \\
0 & \mathrm{~A}_{2}
\end{array}\right]\left[\begin{array}{c}
\alpha \hat{\boldsymbol{\psi}} \\
\ell \mathrm{A}_{2}^{-1} \boldsymbol{\phi}_{2}
\end{array}\right]=\left[\begin{array}{c}
\ell \alpha \hat{\boldsymbol{\psi}} \\
\ell \boldsymbol{\phi}_{2}
\end{array}\right]=\ell \boldsymbol{\phi} .
$$

As the projections $\mathrm{P}_{1}$ and $\mathrm{P}_{2}$ are analytic and $\mathrm{A}_{2}$ is bounded with analytic inverse, $\boldsymbol{\psi}$ is analytic.

Remark. The analyticity of $\boldsymbol{\psi}$ and formula (B7) for the calculation of the scattered fields imply immediately the analyticity of the transmission amplitude $b=b(\kappa, \omega)$ and the reflection amplitude $a=(\kappa, \omega)$ that formed a cornerstone of our analysis in Sec. II.

Any significant field amplitude enhancement should come from the first component of the field $\boldsymbol{\psi}$ and should be measured by the ratio $|\alpha / \ell|$; the second component is of order $O(\ell)$ as is the incident field. If $\alpha$ has a nonzero value when evaluated at $k=0$ and $\omega=\omega_{0}$, the ratio blows up like the reciprocal of the $|\ell|$ as $(\kappa, \omega) \rightarrow\left(0, \omega_{0}\right)$; there is singular field enhancement irrespective of how the limit is taken. This is in disagreement with results in the cases that we have tested numerically [13], including the example we discussed in Sec. II. Indeed, upon illumination of the structures by a plane wave with the value of $\kappa$ fixed at 0 and with $\omega$ close to $\omega_{0}$, no significant field enhancement in the structure is observed. As a result, we assume that $\alpha(0, \omega)=0$. [This means that the source field contains no component of $\hat{\boldsymbol{\psi}}$. The source trace $\boldsymbol{\phi}$ is in the image of $\mathrm{A}$ at $\left(\kappa_{0}=0, \omega_{0}\right)$, and the scattering problem with source trace $\boldsymbol{\phi}$ has a steady-state solution at this pair $\left.\left(\kappa_{0}, \omega_{0}\right).\right]$

We adopt as the generic expansion of $\alpha$ in the vicinity of $\left(0, \omega_{0}\right)$ (recall that $\left.\varpi=\omega-\omega_{0}\right)$

$$
\alpha=\beta_{1} \kappa+\beta_{2} \varpi+\cdots .
$$

Using this form for $\alpha$ and Eq. (6) for $\ell$, we obtain

$$
\frac{\alpha}{\ell}=\frac{\beta_{1} \kappa+\beta_{2} \varpi+\cdots}{\left(\varpi+s_{1} \kappa^{2}\right)+i s_{2} \kappa^{2}+\cdots}\left(\frac{1}{s_{0} e^{i \theta_{1}}}+\cdots\right),
$$

in which $s=s_{1}+s_{2} i$. At normal incidence $(\kappa=0)$, the form we have assumed for $\alpha$ gives us

$$
\mathcal{A} \sim\left|\frac{\alpha}{\ell}\right| \rightarrow \operatorname{const}(\varpi \rightarrow 0, \kappa=0),
$$

which agrees with the numerical observation of no resonant enhancement in this regime.

We now show how the forms of $\alpha$ and $\ell$ lead to a prediction of the leading-order behavior of resonant enhancement within the vicinity of the transmission anomaly (the "spike" in Fig. 3), and then we compare this prediction with numerical data. To this end, let $\kappa$ be a small positive number and allow $\omega$ to range over real values near $\omega_{0}$ (so that $\varpi$ ranges over real values near zero), which corresponds to scattering by harmonic plane-wave sources. The magnitude of the denominator in $\alpha / \ell$ is smallest when $\varpi+s_{1} \kappa^{2} \approx 0$; the corresponding value of $\omega\left(\omega \approx \omega_{0}-s_{1} \kappa^{2}\right)$ lies between the lower and upper peaks of the spike in the transmission graph (Fig. 3 ) for the given value of $\kappa$. This is the real value of $\varpi$ such

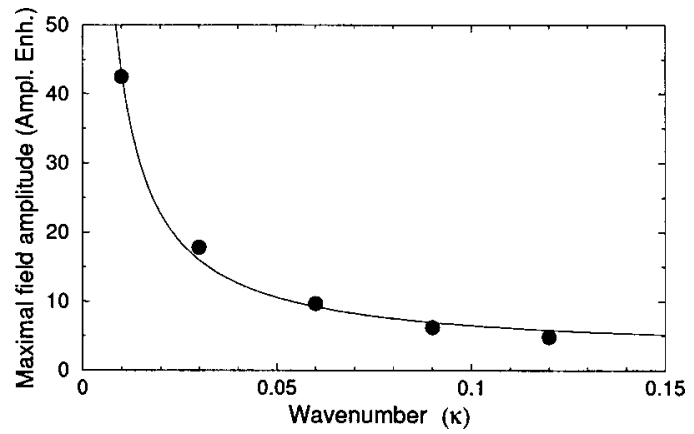

FIG. 6. The solid dots represent numerically calculated maximal values of (the boundary trace of) the total field produced by an incident plane wave of amplitude 1 at various values of $\kappa$ (corresponding to different angles of incidence) at the frequency of optimal enhancement. The solid curve is the best fit of the numerical data to the theoretical form $c_{1} / \kappa+c_{2}$. Here, $c_{1}=0.4053$ and $c_{2}$ $=2.478$.

that $(\kappa, \varpi)$ lies closest to the point $\varpi \approx-\left(s_{1}+s_{2} i\right) \kappa^{2}$ on the dispersion relation (ignoring terms of order $\kappa^{3}$ ). To see the response to an incident plane wave at this optimal frequency, we put

$$
\varpi=-s_{1} \kappa^{2}, \text { or } \omega=\omega_{0}-s_{1} \kappa^{2},
$$

and obtain for the amplitude enhancement $\mathcal{A}$

$$
\mathcal{A}=\left|\frac{\alpha}{\ell}\right| \approx \frac{1}{\kappa}\left|\frac{\beta_{1}-\beta_{2} s_{1} \kappa+\cdots}{i s_{0} s_{2}+\cdots}\right|,
$$

so that $\mathcal{A}$ has the asymptotic form

$$
\mathcal{A} \sim \frac{c_{1}}{\kappa}+c_{2}+\cdots\left(\varpi=-s_{1} \kappa^{2}, \kappa \rightarrow 0\right) .
$$

Figure 6 shows a numerical confirmation of this $1 / \kappa$ law for the field amplitude in the structure. For various values of $\kappa$, the scattering experiment is simulated (using the boundary-integral equations) at the optimal value $\varpi=-s_{1} \kappa^{2}$, and the results are plotted against the best fit of the form (14), using the first two terms. We use the maximal value of the total field $\boldsymbol{\psi}$ to estimate $\mathcal{A}$ numerically. The lower image in Fig. 5 shows a contour plot of the amplitude of the total field produced by a plane-wave source field at the optimal frequency $\omega \approx \omega_{0}-s_{1} \kappa^{2}$ for $\kappa=0.12$.

The constant in Eq. (14) depends on $s_{2}$, as well as on $s_{0}, \beta_{1}$, and the choice of eigenvector $\hat{\boldsymbol{\psi}}$ for A. The dependence on $s_{2}$ is particularly significant, as $s_{2}$ determines the imaginary part of the complex point $\varpi \approx-\left(s_{1}+i s_{2}\right) \kappa^{2}$ on the dispersion relation. The corresponding complex guided mode is a "resonance" for the structure. It grows spatially, as $|z| \rightarrow \infty$, but it decays exponentially in time. It is understood as a model for "leaky" or "quasiguided" modes (see, for example, $[12,8])$. We see from our analysis how the imaginary part of the complex mode affects scattering enhancement at nearby real values of the frequency, particularly at the real part $-s_{1} \kappa^{2}$ of the complex frequency $-\left(s_{1}+s_{2} i\right) \kappa^{2}$. The constant in Eq. (14) is inversely proportional to $s_{2}$. 


\section{ACKNOWLEDGMENTS}

S.P.S. was supported by LA Board of Regents Grant No. LEQSF (2003-06)-RD-A-14. S.V. was supported by NSF Grant No. DMS-0207262.

\section{APPENDIX A: SCATTERING AND GUIDED MODES}

We present the mathematical theory that provides the foundations for the local analytic connection of the scattering states to the slab modes (by means of the field $\psi$ ), which we used in the previous section.

We denote the vector of all electric and magnetic field components with the time-dependent field $\psi(x, y, z) e^{-i \omega t}$, where the spatial part $\psi$ has the Bloch form $\psi$ $=\tilde{\psi}(x, y, z) e^{i\left(\kappa_{1} x+\kappa_{2} y\right)}$, and $\tilde{\psi}$ is $2 \pi$-periodic in $x$ and $y$. We use boundary-integral representations to pose the scattering problem. The scattered and guided modes are specified in terms of the traces (values of the tangential components) of these fields on the interface between contrasting materials composing the slab within a unit cell. The trace of a source field alone is sufficient to determine the trace of the scattered field, which, in turn, determines the total field in space. Details on the integral equations are given in Appendix B.

The integral representations give rise to an explicit bounded integral operator A [Eqs. (B3), (B4a), (B4b), (B6a), and (B6b)] having the following property. If a source field $\phi$, scattered off the slab, produces the total field $\psi$, then the respective field traces $\boldsymbol{\phi}$ of the source field and $\psi$ of the total field satisfy the governing integral equation $\mathrm{A} \boldsymbol{\psi}=\boldsymbol{\phi}$. The integral operator A depends on the geometry, on the frequency $\omega$, and on the Bloch wave vector $\kappa=\left\langle\kappa_{1}, \kappa_{2}\right\rangle$. It involves layer potentials using the pseudoperiodic radiating Green's function $G$,

$$
\begin{aligned}
G(x, y, z)= & -\frac{1}{8 \pi^{2}} \sum_{m, n=-\infty}^{\infty} \frac{e^{\gamma_{m n}|z|}}{\gamma_{m n}} e^{i\left(m+\kappa_{1}\right) x+i\left(n+\kappa_{2}\right) y}, \\
& \nabla^{2} G+\epsilon \mu \omega^{2} G=-\delta \text { in } \mathcal{S}
\end{aligned}
$$

where $\mathcal{S}$ is one period of the structure, $\mathcal{S}=\{(x, y, z): 0 \leqslant x$ $\leqslant 2 \pi, 0 \leqslant y \leqslant 2 \pi,-\infty<z<\infty\}, \quad \gamma_{m n}^{2}=-\epsilon \mu \omega^{2}+\left(m+\kappa_{1}\right)^{2}+(n$ $\left.+\kappa_{2}\right)^{2} \neq 0$, and $\delta$ is the Dirac delta function centered at the origin. $\epsilon$ is the electric permittivity and $\mu$ is the magnetic permeability exterior to the slab. For penetrable objects, the Green function involving the interior coefficients also enters the operator A. For real values of $\epsilon \mu \omega^{2}$ and $\boldsymbol{\kappa}$, the sign of $\gamma_{m n}$ is taken so that the Fourier harmonics are outgoing $\left(\gamma_{m n}\right.$ positive imaginary) or decaying $\left(\gamma_{m n}\right.$ negative real). The values of $\gamma_{m n}$ are continued analytically as complex functions of $\omega$ and $\boldsymbol{\kappa}$. All but a finite number of harmonics decay as $|z|$ $\rightarrow \infty$. The generalized outgoing condition on a field is that it have an expansion in Fourier harmonics of the outgoing fundamental solution $G$ for sufficiently large values of $|z|$,

$$
\psi \sim \sum_{m, n} c_{m n}^{ \pm} e^{i\left(m+\kappa_{1}\right) x+i\left(n+\kappa_{2}\right) y} e^{ \pm \gamma_{m n} z}(z \rightarrow \pm \infty),
$$

where $c_{m n}$ are constant vectors. This condition includes fields that decay as $|z| \rightarrow \infty$, which are characterized by the condition $c_{m n}^{ \pm}=0$ for all propagating harmonics.
Analytic continuation in the complex variables $\boldsymbol{\kappa}$ and $\omega$ allows a unification of the scattering states with guided slab modes, which are represented by sourceless nonzero solutions of the integral equation $(\mathrm{A} \psi=0)$, that is, solutions at pairs $(\boldsymbol{\kappa}, \omega)$ for which the operator A has a zero eigenvalue. When $\omega$ is real-valued, the slab mode is a physical guided Bloch mode, or a bound state; otherwise it is a quasimode, or leaky mode. In the latter case, we have shown that, if $\omega$ has a nonzero imaginary part, the field $\psi$ grows exponentially away from the slab and decays in time [9] (a fact that is easy to see for solid slabs with no periodic structure [12]). The $(\boldsymbol{\kappa}, \omega)$ pairs that support guided modes of any of the two types satisfy the dispersion relation $\operatorname{det}(\mathrm{A}(\boldsymbol{\kappa}, \omega))=0$. The integral operator A is a Fredholm operator of the second kind; it is stably invertible except at discrete eigenvalues, its (Fredholm) determinant is well defined, and it depends analytically on $(\boldsymbol{\kappa}, \omega)$.

\section{APPENDIX B: BOUNDARY INTEGRAL EQUATIONS}

We present here the equations that relate the traces of the total electromagnetic field to traces of the source fields on the interface between contrasting materials. Derivations of these equations can be found in various sources, including [17-19,9]. We present them here as Fredholm integral equations of the second kind,

$$
\mathrm{A} \boldsymbol{\psi}=\boldsymbol{\phi}, \quad \mathrm{A}=(\mathrm{I}+\mathrm{C})
$$

in which $\boldsymbol{\psi}$ is a vector containing boundary values of the "unknown" total field and $\boldsymbol{\phi}$ is a vector containing boundary values of the "known" source fields. I is the identity operator and $\mathrm{C}$ is a compact integral operator involving layer potentials of periodic fundamental solutions (Green's functions) for the Helmholtz equation.

Let $D \in \mathbb{R}^{3}$ denote one cell of a slab structure that is periodic in $x$ and $y$ and bounded in $z$, with boundary $\partial D$ and outward pointing normal vector $n(r)$ at $r \in \partial D$. We suppose that the medium exterior to $D$ is lossless with dielectric coefficient $\epsilon_{0}$ and magnetic coefficient $\mu_{0}$. We make use of a pseudoperiodic radiating fundamental solution (Green's function) $G_{0}$ of the Helmholtz equation with frequency $\omega$. Thus, $G_{0}$ satisfies

$$
\begin{aligned}
& \left(\partial_{x x}+\partial_{y y}+\partial_{z z}+\epsilon_{0} \mu_{0} \omega^{2}\right) G_{0}(x, y, z) \\
& =-e^{i\left(\kappa_{1} x+\kappa_{2} y\right)} \sum_{j, k=-\infty}^{\infty} \delta(x-2 j \pi, y-2 k \pi, z),
\end{aligned}
$$

where $\delta$ is the Dirac delta function with unit strength at the origin, as well as the pseudoperiodic condition

$$
G_{0}(x, y, z)=e^{i\left(\kappa_{1} x+\kappa_{2} y\right)} \widetilde{G}_{0}(x, y, z),
$$

where $\widetilde{G}_{0}$ has period $2 \pi$ in $x$ and $y$, and the radiating condition that all nondecaying Fourier harmonics are outgoing 
as $z \rightarrow \pm \infty$. The Fourier form of $G_{0}$ is

$$
G_{0}(x, y, z)=-\frac{1}{8 \pi} \sum_{n, m=-\infty}^{\infty} \frac{e^{\gamma_{m n}|z|}}{\gamma_{m n}} e^{i\left(m+\kappa_{1}\right) x+i\left(n+\kappa_{2}\right) y},
$$

in which

$$
\gamma_{m n}^{2}=-\mu_{0} \epsilon_{0} \omega^{2}+\left(m+\kappa_{1}\right)^{2}+\left(n+\kappa_{2}\right)^{2} .
$$

It is assumed that $\mu_{m n} \neq 0$ for all pairs $(m, n)$. We take $i \gamma_{m n}$ $<0$ for the finite number of propagating Fourier harmonics, so that they are outgoing as $|z| \rightarrow \infty$, and $\gamma_{m n}<0$ so that the rest of the harmonics decay as $|z| \rightarrow \infty$. We denote by $G_{1}$ the Green's function with $\epsilon_{0}$ and $\mu_{0}$ replaced by $\epsilon_{1}$ and $\mu_{1}$.

Let $(E, H)$ be the total field resulting from the scattering of free Maxwell source fields by the structure, and denote the tangential traces of the total field, for $r \in D$, by

$$
\begin{gathered}
j(r)=-n(r) \times H(r), \\
m(r)=n(r) \times E(r) .
\end{gathered}
$$

If the structure is a perfect metal, then the electric field is zero on $\partial D$ and we allow an exterior source field $\left(E_{\text {so }}^{\text {ext }}, H_{\mathrm{so}}^{\text {ext }}\right)$. In this case, we have an equation for the trace of the total magnetic field for $r \in \partial D$,

$$
j(r)+\int_{\partial D} n(r) \times\left[j\left(r^{\prime}\right) \times \nabla G_{0}\right] d S\left(r^{\prime}\right)=-n(r) \times H(r) .
$$

In the case that the structure is a lossless material with dielectric coefficient $\epsilon_{1}$ and magnetic coefficient $\mu_{1}$, we must make use of the fundamental solution $G_{1}$ for the interior, obtained by replacing the exterior by the interior material coefficients in the expressions for $\gamma_{m n}$ that appear in Green's function (B2). We allow an additional source $\left(E_{\mathrm{so}}^{\text {int }}, H_{\mathrm{so}}^{\mathrm{int}}\right)$ emanating from the interior. Denote the averages of the interior and exterior coefficients by

$$
\bar{\epsilon}=\frac{\epsilon_{0}+\epsilon_{1}}{2}, \quad \bar{\mu}=\frac{\mu_{0}+\mu_{1}}{2},
$$

and form the following combinations of tangential traces of the source fields on $\partial D$ :

$$
\begin{gathered}
q_{1}=\frac{\mu_{0}}{\bar{\mu}}\left(-n \times H_{\mathrm{so}}^{\mathrm{e}}\right)+\frac{\mu_{1}}{\bar{\mu}}\left(-n \times H_{\mathrm{so}}^{\mathrm{i}}\right), \\
q_{2}=\frac{\epsilon_{0}}{\bar{\epsilon}}\left(n \times E_{\mathrm{so}}^{\mathrm{e}}\right)+\frac{\epsilon_{1}}{\bar{\epsilon}}\left(n \times E_{\mathrm{so}}^{\mathrm{i}}\right) .
\end{gathered}
$$

The following integral equations hold, where $r \in \partial D$ :

$$
\begin{aligned}
j(r) & -\frac{1}{\bar{\mu}} \int_{\partial D} n(r) \times\left[j\left(r^{\prime}\right) \times \nabla\left(\mu_{1} G_{1}-\mu_{0} G_{0}\right)\right] d S\left(r^{\prime}\right) \\
& +\frac{i}{\omega \bar{\mu}} \int_{\partial D}\left[n(r) \times m\left(r^{\prime}\right)\right]\left(k_{1}^{2} G_{1}-k_{0}^{2} G_{0}\right) d S\left(r^{\prime}\right) \\
& +\frac{i}{\omega \bar{\mu}} \int_{\partial D} n(r) \times\left\{\left[m\left(r^{\prime}\right) \cdot \nabla\right] \nabla\left(G_{1}-G_{0}\right)\right\} d S\left(r^{\prime}\right)
\end{aligned}
$$

$$
=q_{1}(r)
$$

$$
\begin{aligned}
m(r) & -\frac{1}{\bar{\epsilon}} \int_{\partial D} n(r) \times\left[m\left(r^{\prime}\right) \times \nabla\left(\epsilon_{1} G_{1}-\epsilon_{0} G_{0}\right)\right] d S\left(r^{\prime}\right) \\
& -\frac{i}{\omega \bar{\epsilon}} \int_{\partial D}\left[n(r) \times j\left(r^{\prime}\right)\right]\left(k_{1}^{2} G_{1}-k_{0}^{2} G_{0}\right) d S\left(r^{\prime}\right) \\
& -\frac{i}{\omega \bar{\epsilon}} \int_{\partial D} n(r) \times\left\{\left[j\left(r^{\prime}\right) \cdot \nabla\right] \nabla\left(G_{1}-G_{0}\right)\right\} d S\left(r^{\prime}\right)=q_{2}(r),
\end{aligned}
$$

in which $k_{0}^{2}=\epsilon_{0} \mu_{0} \omega^{2}$ and $k_{1}^{2}=\epsilon_{1} \mu_{1} \omega^{2}$. All of the Green functions and their derivatives are evaluated at $\left(r-r^{\prime}\right)$.

Having solved for $j$ and $m$ on $\partial D$, one then computes $(E, H)$ for $r$ off of $\partial D$ by using standard integral representation formulas.

In the two-dimensional reduction, in which the structure is invariant in the $x$ direction, the fields can be decomposed into two polarizations, in which the electric and magnetic field, respectively, is pointing out of the $y z$ plane $\left(E_{x}\right.$ and $H_{x}$ fields, respectively). $D$ now denotes the two-dimensional cross section of the structure in the $y z$ plane. We make use of the two-dimensional fundamental solution (we reuse the notation $G_{0}$ ),

$$
G_{0}(y, z)=-\frac{1}{4 \pi} \sum_{m=-\infty}^{\infty} \frac{1}{\gamma_{m}} e^{\gamma_{m}|z|} e^{i\left(m+\kappa_{1}\right) y}
$$

in which

$$
\gamma_{m}^{2}=-\mu_{0} \epsilon_{0} \omega^{2}+\left(m+\kappa_{1}\right)^{2}
$$

Let $u$ denote the out-of-plane field component on $\partial D$, and $\partial u / \partial n$ the limiting value from the exterior of $D$ of its normal derivative on $\partial D$. Set $\nu=\mu_{1} / \mu_{0}$ and $\sigma=\left(2 \mu_{0}\right) /\left(\mu_{0}+\mu_{1}\right)$ in the $E_{x}$ case, and $\nu=\epsilon_{1} / \epsilon_{0}$ and $\sigma=\left(2 \epsilon_{0}\right) /\left(\epsilon_{0}+\epsilon_{1}\right)$ in the $H_{x}$ case. For $r \in \partial D$, set

$$
\begin{gathered}
p_{1}=u_{\mathrm{so}}^{\mathrm{e}}(r)+u_{\mathrm{so}}^{\mathrm{i}}(r), \\
p_{2}=\sigma\left(\frac{\partial u_{\mathrm{so}}^{\mathrm{e}}(r)}{\partial n}+\frac{\partial u_{\mathrm{so}}^{\mathrm{i}}(r)}{\partial n}\right) .
\end{gathered}
$$

The following integral equations hold, where $r \in \partial D$ :

$$
\begin{aligned}
u(r) & +\int_{\partial D} \frac{\partial\left(G_{1}-G_{0}\right)}{\partial n\left(r^{\prime}\right)} u\left(r^{\prime}\right) d s\left(r^{\prime}\right) \\
& -\int_{\partial D}\left(\nu G_{1}-G_{0}\right) \frac{\partial u}{\partial n\left(r^{\prime}\right)}\left(r^{\prime}\right) d s\left(r^{\prime}\right)=p_{1}(r),
\end{aligned}
$$




$$
\begin{aligned}
\frac{\partial u(r)}{\partial n} & +\sigma \int_{\partial D} \frac{\partial^{2}\left(G_{1}-G_{0}\right)}{\partial n(r) \partial n\left(r^{\prime}\right)} u\left(r^{\prime}\right) d s\left(r^{\prime}\right) \\
& -\sigma \int_{\partial D} \frac{\partial\left(\nu G_{1}-G_{0}\right)}{\partial n(r)} \frac{\partial u}{\partial n\left(r^{\prime}\right)} d s\left(r^{\prime}\right)=p_{2}(r) .
\end{aligned}
$$

In these equations, $\partial / \partial n\left(r^{\prime}\right)$ refers to differentiation of $G_{0,1}\left(r-r^{\prime}\right)$ with respect to the variable $r^{\prime}$ in the direction of $n\left(r^{\prime}\right)$. Once the field and its normal derivative are determined on the boundary, one computes the scattered field at a point $r$ in the exterior to $D$ by the Green formula

$$
u(r)=\int_{\partial D}\left(\frac{\partial G_{0}\left(r-r^{\prime}\right)}{\partial n} u\left(r^{\prime}\right)-G_{0}\left(r-r^{\prime}\right) \frac{\partial u}{\partial n}\left(r^{\prime}\right)\right) d s\left(r^{\prime}\right),
$$

and in the interior by a similar formula using $G_{1}$.
[1] T. W. Ebbesen, H. J. Lezec, H. F. Ghaemi, T. Thio, and P. Wolff, Nature (London) 391, 667 (1998).

[2] T. J. Kim, T. Thio, T. W. Ebbesen, D. E. Grupp, and H. J. Lezec, Opt. Lett. 24, 266 (1999).

[3] A. Krishnan, T. Thio, T. Kim, H. Lezec, T. Ebbesen, P. Wolff, J. Pendry, L. Martin-Moreno, and F. Garcia-Vidal, Opt. Commun. 200, 1 (2001).

[4] L. Martin-Moreno, F. J. Garcia-Vidal, H. J. Lezec, K. M. Pellerin, T. Thio, J. B. Pendry, and T. W. Ebbesen, Phys. Rev. Lett. 86, 1114 (2001).

[5] J. A. Porto, F. J. García-Vidal, and J. B. Pendry, Phys. Rev. Lett. 83, 2845 (1999).

[6] M. M. J. Treacy, Appl. Phys. Lett. 75, 606 (1999).

[7] H. F. Ghaemi, T. Thio, D. E. Grupp, T. W. Ebbesen, and H. J. Lezec, Phys. Rev. B 58, 6779 (1998).

[8] S. G. Tikhodeev, A. L. Yablonskii, E. A. Muljarov, N. A. Gippius, and T. Ishihara, Phys. Rev. B 66, 045102 (2002).

[9] S. P. Shipman and S. Venakides, SIAM J. Appl. Math. 64, 322 (2003).

[10] H. J. Lezec, A. Degiron, E. Devaux, R. A. Linke, L. Martin-
Moreno, F. J. Garcia-Vidal, and T. Ebbesen, Science 297, 820 (2002).

[11] S. Bains, OE Rep. No. 195 (2000).

[12] H. Haus and D. Miller, IEEE J. Quantum Electron. 22, 310 (1986).

[13] M. A. Haider, S. P. Shipman, and S. Venakides, SIAM J. Appl. Math. 62, 2129 (2002).

[14] E. V. Evans and C. M. Linton, Q. J. Mech. Appl. Math. 46, 253 (1993).

[15] A. I. Markushevich, Theory of Functions of a Complex Variable (Chelsea Publishing Company, New York, 1985).

[16] T. Kato, Perturbation Theory for Linear Operators (SpringerVerlag, Berlin, 1976).

[17] C. Muller, Foundations of the Mathematical Theory of Electromagnetic Waves (Springer-Verlag, Berlin, 1969).

[18] D. L. Colton and R. Kress, Integral Equation Methods in Scattering Theory (Krieger Publishing Company, Malabar, Florida, 1992).

[19] J.-C. Nédélec, Acoustic and Electromagnetic Equations (Springer-Verlag, New York, 2001). 